\title{
PRognostic factor of Early Death In phase II Corsmank Trials or the end of 'sufficient life expectancy' as an inclusion criterion? (PREDIT model)
}

Thomas Grellety ${ }^{1,2}$, Sophie Cousin ${ }^{1}$, Louis Letinier²,3, Pauline Bosco-Lévy ${ }^{2,3}$, Stéphanie Hoppe ${ }^{3}$, Damien Joly², Nicolas Penel ${ }^{4}$, Simone Mathoulin-Pelissier ${ }^{2,3,5}$ and Antoine Italiano ${ }^{1 *}$

\begin{abstract}
Background: Optimizing patient selection is a necessary step to design better clinical trials. 'Life expectancy' is a frequent inclusion criterion in phase II trial protocols, a measure that is subjective and often difficult to estimate. The aim of this study was to identify factors associated with early death in patients included in phase II studies.

Methods: We retrospectively collected medical records of patients with advanced solid tumors included in phase II trials in two French Comprehensive Cancer Centers (Bordeaux, Center 1 set; Lille, Center 2 set). We analyzed patients' baseline characteristics. Predictive factors associated with early death (mortality at 3 months) were identified by logistic regression. We built a model (PREDIT, PRognostic factor of Early Death In phase II Trials) based on prognostic factors isolated from the final multivariate model.

Results: Center 1 and 2 sets included 303 and 227 patients, respectively. Patients from Center 1 and 2 sets differed in tumor site, urological (26\% vs $15 \%)$ and gastrointestinal (18\% vs $28 \%)$ and in lung metastasis incidence (10 \% vs $49 \%$ ). Overall survival (OS) at 3 months was $88 \%$ (95\% Cl [83.5; 91.0], Center 1 set) and $91 \%$ (95\% Cl [86.7; 94. 2], Center 2 set). Presence of a 'life expectancy' inclusion criterion did not improve the 3-month OS ( $\mathrm{HR} 0.6,95 \% \mathrm{Cl}$ $[0.2 ; 1.2], p=0.2325)$. Independent factors of early death were an ECOG score of 2 (OR 13.3, 95\%Cl [4.1; 43.4]), hyperleukocytosis (OR 5.5, $95 \% \mathrm{Cl}[1.9 ; 16.3])$ and anemia (OR 2.8, $95 \% \mathrm{Cl}[1.1 ; 7.1])$. Same predictive factors but with different association levels were found in the Center 2 set. Using the Center 1 set, ROC analysis shows a good discrimination to predict early death (AUC: 0.89 at 3 months and 0.86 at 6 months).

Conclusions: Risk modeling in two independent cancer populations based on simple clinical parameters showed that baseline ECOG of 2, hyperleukocytosis and anemia are strong early-death predictive factors. This model allows identifying patients who may not benefit from a phase II trial investigational drug and may, therefore, represent a helpful tool to select patients for phase II trial entry.
\end{abstract}

Keywords: Phase II trial, Early death, Prognostic factors, "life expectancy" criterion, Drug trials

\footnotetext{
*Correspondence: A.ttaliano@bordeaux.unicancer.fr

'Department of Medical Oncology, Institut Bergonié, Comprehensive Cancer

Centre Bordeaux, 229 cours de l'Argonne, 33076 Bordeaux, France

Full list of author information is available at the end of the article
} 


\section{Background}

Phase II trials in oncology are an essential part in anticancer drug development as they provide relevant data regarding toxicity and proof of efficacy. These assessments are necessary to make the 'go or no-go' decision before starting large controlled randomized phase III trials [1]. In oncology, there are more phase II (45\% vs $23 \%$ ) but fewer phase III (13\% vs $23 \%$ ) trials than in other specialties [2]. Phase II to phase III represents the riskiest transition point of the drug development pathway $[3,4]$, as proven by the very high attrition rate between a successful phase II and the subsequent phase III trial. Enhancing the overall quality of phase II trials is therefore critical for drug development, and could benefit from changes at several levels, from the use of randomization in the study design [5] to the improvement in the quality of publication [6]. Furthermore, there is a need to rethink the selection of large numbers of patients for phase II trials that raise ethical and cost questions. Indeed, patient selection has been recognized as being of upmost importance in the design of clinical trials [7]. Although many efforts have been made in phase I trials wherein a careful patient selection likely increases the benefit of the trial to patients, no such initiative has been taken for phase II trials. Similarly, there is an increase in the average number of inclusion criteria for phase II trials, such as 'sufficient life expectancy' at screening [8]. Life expectancy is difficult to estimate in clinical practice and depends on the physician's consideration, making it not only irreproducible but also insufficient to predict any benefit for the patient, as most patients enroll with a hope for therapeutic benefit [9]. Ethical consideration should therefore lead physicians to include patients only in cases of potential benefit from the investigational drug. This would require identifying those patients that would survive long enough for the investigational treatment to be effective. Despite the crucial role of phase II trials in drug development, no tool has been published that allows a better selection of patients based on their prognostic. The aim of this pilot study is to develop a model to identify prognostic factors of early death in adult cancer patients included in oncology phase II trials based on two sets of patients from two French Comprehensive Cancer Centers. Relevant prognostic factors will help investigators identify participants unsuitable for such studies.

\section{Methods}

\section{Selection of patients}

The first patient set (Center 1 set) included all patients involved in phase II clinical trials at the Institut Bergonié, Comprehensive Cancer Center (Bordeaux, France), between January 2008 and December 2012. We selected all trials investigating anticancer drugs and having included adults (aged 18 or older) with advanced or metastatic solid tumors. Trials investigating supportive care, surgical procedures or radiotherapy were excluded. Patients had received at least one dose of the investigational agent. The second set (Centre 2 set) was from the Oscar Lambret Cancer Center (Lille, France), with all patients included in a phase II clinical trial between January 2011 and July 2014 that met the same criteria.

For each patient, retrospective baseline data were recorded at inclusion in the phase II trial: age, gender, body mass index (BMI), ECOG performance status, histology, number and sites of metastasis, treatment type, biological data (serum albumin, Lactate Dehydrogenase (LDH), platelets, leukocyte and lymphocyte counts, hemoglobin level, sodium, potassium and calcium level, alkaline phosphatase, alanine and aspartate transaminase and c-reactive protein). Furthermore, for each patient we recorded the date of inclusion, and date and cause of study withdrawal.

The following data regarding the design of the clinical trials were extracted from each protocol: presence of a "life expectancy" inclusion criterion, randomized trial (Yes vs No), number of previous treatment lines authorized and nature of the promoter (academic vs industrial). Study data were collected and managed using REDCap electronic data capture tools [10].

\section{Statistical methods}

Variables were described using median, mean and extreme values. Categorical variables were classified based on the normal values (for biological variables, BMI). Biological variables were classified as normal, below normal and above normal. Overall survival (OS) was defined as the time from inclusion in a trial to death from any cause. Patients lost during follow-up were censored at their last visit. Survival was estimated using the KaplanMeier method. For our main analysis, we used early deaths, defined as all deaths occurring up to 3 months from inclusion. We also performed a secondary analysis for deaths occurring up to 6 months from inclusion. Three- and six-month' cut-off's were chosen due to their discriminant nature in the detection of prognostic factors. Three months represents the classical cut-off point for the first evaluation of safety and efficacy in clinical trials. It has commonly been used in studies of prognostic factors for patients included in phase I trials $[11,12]$ and is relevant regarding the median overall survival of 9.4 months for patients included in phase II trials, as published in a recent meta-analysis by Schwaederle M. et al. [13].

On the Center 1 set, we performed a logistic model to estimate odds ratio (OR) and $95 \%$ confidence interval (95\% CI) of the association between early death and clinical or biological variables. All variables associated with a significantly increased risk of early death $(p<0.05)$ were 
considered for multivariate analysis. Variables such as age, sex and tumor localization were included in all models due to clinical relevance. Selection of variables for the multivariate model was performed following a step-bystep forward strategy. In order to limit the number of variables in the final multivariate model, clinical and laboratory variables were first selected in two separate specific multivariate models using stepwise logistic approach. Each clinical and biological variable selected in their respective multivariate model was entered into a third and final model before adjusting for age, sex and tumor localization. The threshold of 0.05 for statistical significance was used to maintain the variable in the model. The stringent alpha level allowed limiting the selection to those factors that are relevant from a clinician's perspective.

A model (PREDIT, PRognostic factor of Early Death In phase II Trial) was built with the prognostic factors isolated from the final multivariate model in the Center 1 set. Adequacy was established using the Hosmer \& Lemeshow test. [14]. Discrimination of mortality at 3 and 6 months was evaluated using the receiver operator characteristic area under the curve (AUC). Finally, we performed the same analyses in the Centre 2 set. Statistical analyses were carried out using the SAS software, version 9.3 (SAS Institute, Inc., Cary, NC).

\section{Results}

\section{Characteristics of the trials}

Fifty-one trials were included for analysis in the Center 1 set and 40 in the Center 2 set, with recruitment ranging from one to 31 patients. Patient characteristics are described in Table 1. Twenty-six trials (51\%) in the Center 1 set and 27 trials in the Center 2 set $(68 \%)$ were sponsored by a pharmaceutical company. Most phase II trials were randomized (59 \% in the Center 1 set and $63 \%$ in the Center 2 set). Treatments differed between

Table 1 Characteristics of trials and outcomes for Center 1 and Center 2 sets

\begin{tabular}{|c|c|c|c|c|c|}
\hline \multirow[t]{2}{*}{ Characteristics } & \multicolumn{2}{|c|}{ Center 1 Set $(N=303)$} & \multicolumn{2}{|c|}{ Center 2 Set $(N=227)$} & \multirow[t]{2}{*}{ P Chi2 } \\
\hline & $\overline{N(\%)}$ & Median (Min-Max) & $\overline{N(\%)}$ & Median (Min-Max) & \\
\hline Number of trials & 51 & & 40 & & \\
\hline Patients by trials & & $4(1-31)$ & & $2(1-22)$ & \\
\hline Trial randomization & & & & & 0.13 \\
\hline Yes & $30(59)$ & & $25(63)$ & & \\
\hline No & $21(41)$ & & $15(37)$ & & \\
\hline Trial promotion & & & & & 0.08 \\
\hline Academic & $25(49)$ & & $27(68)$ & & \\
\hline Industrial & $26(51)$ & & $13(32)$ & & \\
\hline Protocol defined treatments & & & & & $<0.001$ \\
\hline Chemotherapy-based regimen & $154(51)$ & & $157(69)$ & & \\
\hline Targeted therapies only (targeted therapies and/or endocrine therapy) & $149(49)$ & & $70(31)$ & & \\
\hline Protocol specified "life expectancy" criterion (per patient) & & & & & 0.003 \\
\hline Yes & $73(24)$ & & $82(36)$ & & \\
\hline No & $230(76)$ & & $145(64)$ & & \\
\hline Treatment duration (months) & & $4(0-44)$ & & $4(0-49)$ & \\
\hline Reason for discontinuing trial & & & & & $<0.001$ \\
\hline Progression & $198(65)$ & & $136(60)$ & & \\
\hline Programmed end of the trial & $42(14)$ & & $6(3)$ & & \\
\hline Toxicity & $32(10)$ & & $37(16)$ & & \\
\hline Death & $12(4)$ & & $2(1)$ & & \\
\hline Patient Retrial & $8(3)$ & & $9(4)$ & & \\
\hline Other & $8(3)$ & & $18(8)$ & & \\
\hline NA & $3(1)$ & & $19(8)$ & & \\
\hline Median follow-up (months) & & $17(0-77)$ & & $12(0-50)$ & \\
\hline Three-month mortality rate & $37(12)$ & & $20(9)$ & & \\
\hline Six-month mortality rate & $59(20)$ & & $41(18)$ & & \\
\hline
\end{tabular}

Abreviations: NA not available 
Table 2 Clinical and biological characteristics at baseline for Center 1 and Center 2 sets

\begin{tabular}{|c|c|c|c|c|c|}
\hline \multirow[t]{2}{*}{ Characteristics at baseline } & \multicolumn{2}{|c|}{ Center 1 Set $(N=303)$} & \multicolumn{2}{|c|}{ Center 2 Set $(N=227)$} & \multirow[t]{2}{*}{ PChi2 } \\
\hline & $N(\%)$ & Median (Min-Max) & $N(\%)$ & Median (Min-Max) & \\
\hline Sex & & & & & 0.64 \\
\hline Male & $181(60)$ & & $131(58)$ & & \\
\hline Female & $122(40)$ & & $96(42)$ & & \\
\hline Age & & $62(\mathrm{IQR}, 19)$ & & $60(I Q R, 23)$ & 0.07 \\
\hline$<50$ & $55(18)$ & & $42(19)$ & & \\
\hline $50-65$ & $116(38)$ & & $107(47)$ & & \\
\hline$\geq 65$ & $132(44)$ & & $78(34)$ & & \\
\hline Performance status & & & & & 0.82 \\
\hline ECOG 0-1 & $278(92)$ & & $207(91)$ & & \\
\hline ECOG 2 & $25(8)$ & & $20(9)$ & & \\
\hline BMI $\left(\mathrm{kg} / \mathrm{m}^{2}\right)$ & & $25(16-39)$ & & $26(16-45)$ & $-{ }^{a}$ \\
\hline$<18.5$ & $13(4)$ & & $9(4)$ & & \\
\hline $18.5-25$ & $147(49)$ & & $83(37)$ & & \\
\hline$\geq 25$ & $143(47)$ & & $107(47)$ & & \\
\hline NA & 0 & & $28(12)$ & & \\
\hline Previous treatments & & $1(0-4)$ & & $1(0-6)$ & $<0.001$ \\
\hline 0 & $9(3)$ & & $106(47)$ & & \\
\hline 1 & $185(61)$ & & $67(30)$ & & \\
\hline 2 & $74(24)$ & & $32(14)$ & & \\
\hline 3 & $27(9)$ & & $19(8)$ & & \\
\hline 4 and more & $8(3)$ & & $3(1)$ & & \\
\hline Cancer localization & & & & & 0.01 \\
\hline Sarcomas & $123(40)$ & & $91(40)$ & & \\
\hline Urological & $78(26)$ & & $35(15)$ & & \\
\hline Gastrointestinal & $54(18)$ & & $63(28)$ & & \\
\hline Breast & $29(10)$ & & $20(9)$ & & \\
\hline Other & $19(6)$ & & $18(8)$ & & \\
\hline Involved areas & & & & & 0.55 \\
\hline Loco-regional disease & $18(6)$ & & $19(8)$ & & \\
\hline 1-2 metastatic sites & $223(74)$ & & $164(72)$ & & \\
\hline$\geq 3$ metastatic sites & $62(20)$ & & $44(19)$ & & \\
\hline Hemoglobin level & & & & & 0.08 \\
\hline$<$ Normal & $106(35)$ & & $94(41)$ & & \\
\hline Normal & $196(65)$ & & $126(56)$ & & \\
\hline NA & $1(0.3)$ & & 7 (3) & & \\
\hline Leukocyte level & & & & & 0.18 \\
\hline$<$ Normal & $14(5)$ & & $15(6)$ & & \\
\hline Normal & $255(84)$ & & $171(75)$ & & \\
\hline$>$ Normal & $33(11)$ & & $33(15)$ & & \\
\hline NA & $1(0.3)$ & & $8(4)$ & & \\
\hline Albumin level & & & & & $-{ }^{a}$ \\
\hline$<$ Normal & $25(8)$ & & $87(38)$ & & \\
\hline
\end{tabular}


Table 2 Clinical and biological characteristics at baseline for Center 1 and Center 2 sets (Continued)

\begin{tabular}{lll}
\hline Normal & $236(78)$ & $111(50)$ \\
NA & $32(11)$ & $28(12)$ \\
LDH level & & $137(60)$ \\
$<$ Normal & $133(44)$ & $51(23)$ \\
Normal & $55(18)$ & $34(15)$ \\
NA & $111(37)$ & $-{ }^{a}$ \\
\hline
\end{tabular}

Abbreviations: BMI body mass index, ECOG Eastern Cooperative Oncology Group, IQR interquartile range, $L D H$ lactate dehydrogenase, NA not available

${ }^{a}$ Due to a non-applicable rate of $10 \%$ or more, BMI, Albumin level and LDH level variables cannot be tested by the chi test

the two sets: whereas the ratio between targeted therapy $(149,49 \%)$ and chemotherapy $(154,51 \%)$ was wellbalanced in the Center 1 set, chemotherapy was more frequently used $(157,69 \%)$ in the Center 2 set.

\section{Patient characteristics}

The Center 1 and Center 2 sets included 303 and 227 patients, respectively. Median age was 62 years (Interquartile Range, 19) in the Center 1 set and 60 (Interquartile Range, 23) years in the Center 2 set. The male to female ratio was similar in both sets (Center 1 set: 1.5 and Center 2 set: 1.4). Primary tumor sites were equally distributed for sarcomas (123, $41 \%$; 91, $40 \%$ ) and breast $(29,10 \%$; $20,9 \%)$ but differed significantly for urological $(78,26 \% ; 35,15 \%)$ and gastrointestinal (54, $19 \%$; 63, $28 \%)$ cancers. There were 241 (80 \%) and 183 $(81 \%)$ patients with two or less metastatic sites in the Center 1 and Center 2 sets, respectively. Occurrence of liver metastases was similar in both groups, (Center 1 set: 106, $35 \%$; Center 2 set: 73, $32 \%$ ) whereas lung metastases were rarer in the Center 1 set (Center 1 set: 30, $10 \%$; Center 2 set: 111, $49 \%$ ); there was more bone and extra-regional lymph nodes involvement in the Center 1 set. Median number of previous lines of treatment was one (Center 1 set: range 0-4; Center set: range 0-6). Clinical and biological values at baseline are described in Table 2.

\section{General description}

Median time to trial discontinuation was 4.0 months in the two sets (range $0-44$ and $0-49$, respectively). Most patients were withdrawn from the study due to disease progression (Center 1 set: 198, $65 \%$; Center 2 set: 136, $60 \%$ ) or to toxicity (Center 1 set: 32, $11 \%$; Center 2 set: $37,16 \%)$. Overall survival at 3 months in the Center 1 and 2 sets were $88 \%(95 \% \mathrm{CI}, 83.5-91.0)$ and $91 \%$ (95\% CI, 86.7-94.2), respectively. Life expectancy was included as an eligibility criterion in 13 trials (73 patients, $24 \%$ ) in the Center 1 set and 19 trials (82 patients, $36 \%$ ) in the Center 2 set. The presence of life expectancy among the inclusion criteria did not improve the 3-month OS in either the Center 1 (hazards ratio [HR] 0.6, $95 \% \mathrm{CI}, 0.2-1.2, P=.23$ ) or the Center 2 (HR $0.7,95 \% \mathrm{CI}, 0.3-2.0, P=.55$ ) set (Fig. 1).

\section{Factors associated with 3-month early deaths}

Results from univariate and multivariate analyses are presented in Table 3 and Additional file 1: Table S1 (onlineonly supplementary material). Factors associated with the 3-month mortality in multivariate analysis in the Center 1 set, after adjustment on age, sex and tumor localization, included an ECOG performance status of 2 (OR 13.3, 95 \% CI, 4.1-43.4), hyperleukocytosis (OR 5.5, 95 \% CI, 1.9-16.3) and anemia (OR 2.8, 95 \% CI, 1.1-7.1). Based on these three factors, we calculated a risk (PREDIT

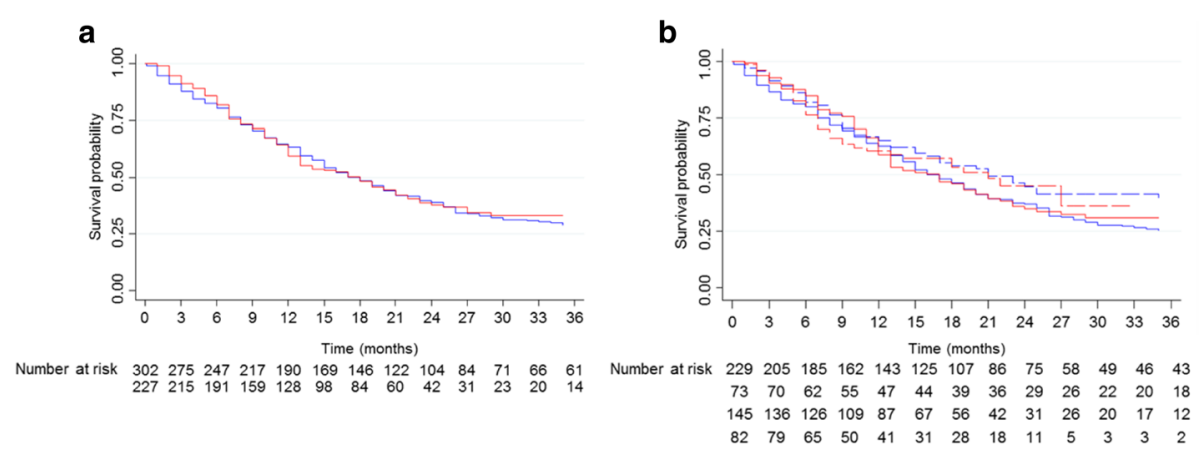

Fig. 1 Overall survival in the two sets. a Overall survival in the Center 1 set (blue) and Center 2 set (red). b Survival depending on the presence (dotted lines) or absence (full lines) of 'life expectancy' criterion for each patient included regarding the respective trial's protocol, in the Center 1set (blue) and Center 2 set (red) 
Table 3 Factors associated with early death at 3 months in the Center 1 set $(N=303)$

\begin{tabular}{|c|c|c|c|c|c|c|c|}
\hline \multirow[b]{2}{*}{ Characteristics at baseline } & \multirow[b]{2}{*}{ Early deaths $N(\%)$} & \multicolumn{3}{|c|}{$\underline{\text { Univariate analysis }}$} & \multicolumn{3}{|c|}{$\underline{\text { Multivariate analysis }}$} \\
\hline & & $\mathrm{OR}$ & $95 \% \mathrm{Cl}$ & P & $\mathrm{OR}$ & $95 \% \mathrm{Cl}$ & $P$ \\
\hline Age & & & & 0.01 & & & 0.21 \\
\hline$<50$ & $12(32)$ & 1 & & & 1 & & \\
\hline$[50-65]$ & $17(46)$ & 0.2 & {$[0.1-0.6]$} & & 1.8 & {$[0.6-5.7]$} & \\
\hline$\geq 65$ & $8(22)$ & 0.6 & {$[0.3-1.4]$} & & 0.7 & {$[0.2-2.4]$} & \\
\hline Sex & & & & 0.17 & & & 0.1500 \\
\hline Female & $11(30)$ & 1 & & & 1 & & \\
\hline Male & $26(70)$ & 1.7 & {$[0.8-3.5]$} & & 2.2 & {$[0.8-6.2]$} & \\
\hline Performance status & & & & $<0.001$ & & & $<0.001$ \\
\hline ECOG 0/1 & $21(57)$ & 1 & & & 1 & & \\
\hline ECOG 2 & $16(43)$ & 21.7 & {$[8.5-54.9]$} & & 13.3 & {$[4.1-43.4]$} & \\
\hline Cancer localization & & & & 0.02 & & & 0.08 \\
\hline Sarcoma & $26(70)$ & 1 & & & 1 & & \\
\hline Gastrointestinal & $4(11)$ & 0.3 & {$[0.1-0.9]$} & & 0.2 & {$[0.0-0.7]$} & \\
\hline Urological & $5(14)$ & 0.3 & {$[0.1-0.7]$} & & 0.3 & {$[0.1-1.2]$} & \\
\hline Breast & $2(5)$ & 0.3 & {$[0.1-1.2]$} & & 0.4 & {$[0.1-1.0]$} & \\
\hline Other & 0 & $<0.1$ & {$[<0.1->999]$} & & $<0.1$ & {$[<0.1->999]$} & \\
\hline Hemoglobin level & & & & $<0.001$ & & & 0.03 \\
\hline Normal & $12(32)$ & 1 & & & 1 & & \\
\hline$<$ Normal & $25(68)$ & 4.8 & {$[2.3-10.0]$} & & 2.8 & {$[1.1-7.1]$} & \\
\hline Leukocyte level & & & & $<0.001$ & & & 0.006 \\
\hline Normal & $22(59)$ & 1 & & & 1 & & \\
\hline$<$ Normal & $1(3)$ & 0.8 & [0.1-6.5] & & 0.4 & {$[0.0-5.4]$} & \\
\hline$>$ Normal & $14(38)$ & 7.8 & [3.4-17.6] & & 5.5 & [1.9-16.3] & \\
\hline
\end{tabular}

Abbreviations: $\mathrm{Cl}$ confidence interval, ECOG Eastern Cooperative Oncology Group, OR odds ratio Bold data reflects significant value

model) for patients included in a phase II trial with the following equation:

Model $=0.4177 \times($ Age $=[50-65[)+0.5950 \times($ Age $=\geq$ $65)+0.7703 x($ Sex $=$ Male $)-0.8658 x($ Cancer localization $=$ Breast $)-1.3116 \times($ Cancer localization $=$ Urogenital) $-13.3383 \times$ (Cancer localization = Other $)$

- $1.7239 x$ (Cancer localization = Gastrointestinal $)+$ $2.5882 x(E C O G=2)-0.8887 \times$ (Leukocyte level $=$ Below the norm $)+1.7050 \times$ (Leukocyte level $=$ Above the norm) $+1.0323 x$ (Hemoglobin level = Below the norm)

Risk calculation revealed a good predictive value for both 3-month and 6-month mortality rates, with AUC values ranging from 0.7 to 0.9 in both sets. More concretely, in the overall population, patients with $0,1,2$ and 3 risk factors had a rate of a 3-month early-death of $2 \%$ (7/292), $14 \%(24 / 175), 38 \%(20 / 53)$ and $60 \%(6 / 10)$ and a rate of 6-month early-death of $7 \%$ (19/292), $28 \%$ (49/ 175), $47 \%$ (25/53) and $70 \%(7 / 10)$, respectively.

\section{Discussion}

Phase II trials are crucial screening tools to assess whether an anti-cancer drug has sufficient activity to warrant further investigation in large, costly phase III trials. In this respect, patients should be selected for such trials in a manner that maximizes the potential to assess the clinical activity of the investigational drug. Patients in poor conditions and with a limited life expectancy are not likely to derive significant benefit from the investigational therapy and inclusion of such patients may preclude valid conclusions about the clinical activity of the drug. Therefore, appropriate assessment of life expectancy is crucial to avoid inclusion of patients who are at higher risk of early death and who have low probability of clinical benefit. Our prognostic factors, ECOG performance status of 2, hyperleukocytosis and anemia, validated in two independent sets, could provide physicians with an objective tool to help in this assessment.

Performance status is a well-known bad prognostic factor in oncology, associated with early death and already described for patients included in phase I trials $[15,16]$. Anemia is very frequent in oncology with a 
prevalence of $39 \%$ at onset of cancer, and $68 \%$ of patients present anemia at least once in the subsequent 6 months [17]. It leads to an overall relative increase in risk of death of $65 \%$ (54-77\%) [18] that can be related to various factors. A decrease in WHO performance score and quality of life are well-known consequences [17] that can limit options for specific cancer treatments. The worst outcomes associated to anemia can also be linked to its biological consequences. As an example, a more aggressive cancer biology is connected to the promotion of hypoxia-inducible factor 1 , which is induced by anemic hypoxia and has been described as a tumor metastasis enhancer [19]. Initial hyperleukocytosis (often associated with neutrophilia) is a frequent event in patients with solid tumors, with an incidence ranging from 4 to $26 \%$ [20], and has been associated with poor outcome in several solid tumor types [21-28]. Indeed, leukocytosis is related to tumor burden $[27,29]$. Additionally, it can be a consequence of processes such as infection and/or inflammation or corticosteroids treatment [29, 30], which can themselves be of bad prognostic.

This preliminary study allowed developing a first model that needs to be validated at the national level. One of the advantages of a two-step procedure is the possibility to evaluate the feasibility on the data collection in the medical record. One of the disadvantages is that preliminary results are not confirmed on a larger population. Besides the limited power, the main limitation of our study lies in its retrospective nature. Prospectively defining a model for patients that would be included in a phase II trial would be very complex, and, as a consequence, only patients who passed screening and received at least one dose of the investigational agent were included in the study (whatever the set). One of the strengths of our study is that the sets originated from two different cancer centers. We identified the same three prognostic factors in the Center 2 set despite differences in the characteristics of the two populations. This demonstrates a strong relevance of these factors to predict early death regardless of the heterogeneity of patients, primary tumor sites, treatments and management strategies. Further work will aim at validating the model in an independent and wider cohort of patients such as a national cancer registry.

\section{Conclusions}

Risk modeling based on simple clinical parameters including hemoglobin level, leukocyte count and ECOG performance status indicated that patients with two or more prognostic factors had a significant risk of early death. Our results clearly suggest that these patients should be considered carefully for inclusion in a phase II clinical trial. Our model may represent a helpful tool in the process of patient selection for phase II trial entry.

\section{Additional file}

Additional file 1: Table S1. Univariate analysis of factors non-associated with 3-month mortality in the multivariate model for the Centre 1 set. (DOCX $25 \mathrm{~kb}$ )

\section{Abbreviations}

AUC: Area under the curve; BMI: Body mass index; Cl: Confidence interval; ECOG: Eastern cooperative oncology group; HR: Hazards ratio; LDH: Lactate dehydrogenase; NA: Not available; OR: Odds ratio; OS: Overall survival

\section{Acknowledgements}

We would like to thank Dr. Jone Iriondo-Alberdi and Dr. Ravi Nookala of Institut Bergonié for the medical writing service.

\section{Funding}

The present study has been funded by the Institut Bergonié, Comprehensive Cancer Center.

\section{Availability of data and materials}

The datasets supporting the conclusions of this article cannot be shared for confidentiality reasons.

\section{Authors' contributions}

Study concept and design: TG, SM-P, Al. Acquisition, analysis or interpretation of data: TG, SC, LL, PB-L, SH, DJ, NP. Drafting of the manuscript: TG, SH, SM-P,

Al. Critical revision of the manuscript for important intellectual content: SC,

$L L, P B-L, D J, N P$. All authors have given final approval of the version to be published. All authors agree to be accountable for all aspects of the work in ensuring that questions related to the accuracy or integrity of any part of the work are appropriately investigated and resolved.

\section{Competing interests}

The authors declare that they have no competing interests.

\section{Consent to publish}

Not applicable.

\section{Ethics approval and consent to participate}

This study was approved by the ethics committees of the Comprehensive Cancer Center Institut Bergonié (Bordeaux, France) and of the Comprehensive Cancer Center Oscar Lambret (Lille, France).

\section{Author details}

${ }^{1}$ Department of Medical Oncology, Institut Bergonié, Comprehensive Cancer Centre Bordeaux, 229 cours de l'Argonne, 33076 Bordeaux, France.

${ }^{2}$ University of Bordeaux, Bordeaux, France. ${ }^{3}$ Clinical and Epidemiological Research Unit Institut Bergonié, Bordeaux, France. ${ }^{4}$ General Oncology Department, Centre Oscar Lambret, Lille, France. ${ }^{5}$ INSERM, CIC1401

Epidemiological unit, Institut Bergonié, Bordeaux, France.

Received: 7 April 2016 Accepted: 26 September 2016

Published online: 04 October 2016

References

1. Roberts TG, Lynch TJ, Chabner BA. The phase III trial in the era of targeted therapy: unraveling the "go or no go" decision. J Clin Oncol. 2003;21:3683-95.

2. Hirsch BR, Califf RM, Cheng SK, et al. Characteristics of oncology clinical trials: insights from a systematic analysis of ClinicalTrials.gov. JAMA Intern Med. 2013;173:972-9.

3. Walker I, Newell H. Do molecularly targeted agents in oncology have reduced attrition rates? Nat Rev Drug Discov. 2009:8:15-6.

4. Zia MI, Siu LL, Pond GR, Chen EX. Comparison of outcomes of phase II studies and subsequent randomized control studies using identical chemotherapeutic regimens. J Clin Oncol. 2005;23:6982-91.

5. Seymour L, Ivy SP, Sargent D, et al. The design of phase II clinical trials testing cancer therapeutics: consensus recommendations from the clinical trial design task force of the national cancer institute investigational drug steering committee. Clin Cancer Res. 2010;16:1764-9. 
6. Grellety T, Petit-Monéger A, Diallo A, et al. Quality of reporting of phase II trials: a focus on highly ranked oncology journals. Ann Oncol. 2014;25:536-41.

7. Kim ES, Bernstein D, Hilsenbeck SG, Chung CH, Dicker AP, Ersek JL, et al. Modernizing Eligibility Criteria for Molecularly Driven Trials. J Clin Oncol. 2015;33:2815-20.

8. Penel N, Clisant S, Lefebvre JL, et al. "Sufficient life expectancy": an amazing inclusion criterion in cancer phase II-III trials. J Clin Oncol. 2009;27:e105.

9. Nurgat ZA, Craig W, Campbell NC, et al. Patient motivations surrounding participation in phase I and phase II clinical trials of cancer chemotherapy. Br J Cancer. 2005;92:1001-5.

10. Harris PA, Taylor R, Thielke R, et al. Research electronic data capture (REDCap)-a metadata-driven methodology and workflow process for providing translational research informatics support. J Biomed Inform. 2009;42:377-81.

11. Arkenau HT, Olmos D, Ang JE, et al. Clinical outcome and prognostic factors for patients treated within the context of a phase I study: the Royal Marsden Hospital experience. Br J Cancer. 2008;98:1029-33.

12. Chau NG, Florescu A, Chan KK, et al. Early mortality and overall survival in oncology phase I trial participants: can we improve patient selection? BMC Cancer. 2011;11:426.

13. Schwaederle $\mathrm{M}$, Zhao $\mathrm{M}$, Lee $\mathrm{JJ}$, et al. Impact of precision medicine in diverse cancers: a meta-analysis of phase II clinical trials. J Clin Oncol. 2015; 33:3817-25

14. Hosmer DW, Lemeshow S. A goodness-of-fit test for the multiple logistic regression model. Commun Stat. 1980;A10:1043-69.

15. Bachelot T, Ray-Coquard I, Catimel G, et al. Multivariable analysis of prognostic factors for toxicity and survival for patients enrolled in phase I clinical trials. Ann Oncol. 2000;11:151-6.

16. Stavraka C, Pinato DJ, Turnbull SJ, et al. Developing an objective marker to optimize patient selection and predict survival benefit in early-phase cancer trials. Cancer. 2014;120:262-70

17. Ludwig H, Van Belle $\mathrm{S}$, Barrett-Lee $\mathrm{P}$, et al. The European Cancer Anaemia Survey (ECAS): a large, multinational, prospective survey defining the prevalence, incidence, and treatment of anaemia in cancer patients. Eur J Cancer. 2004;40:2293-306.

18. Caro JJ, Salas M, Ward A, et al. Anemia as an independent prognostic factor for survival in patients with cancer: a systemic, quantitative review. Cancer. 2001;91:2214-21.

19. Yang MH, Wu MZ, Chiou SH, et al. Direct regulation of TWIST by HIF-1 alpha promotes metastasis. Nat Cell Biol. 2008;10:295-305.

20. Qiu MZ, Xu RH, Ruan DY, et al. Incidence of anemia, leukocytosis, and thrombocytosis in patients with solid tumors in China. Tumour Biol. 2010;31: 633-41.

21. Banerjee $R$, Roxin $G$, Eliasziw $M$, et al. The prognostic significance of pretreatment leukocytosis in patients with anal cancer treated with radical chemoradiotherapy or radiotherapy. Dis Colon Rectum. 2013;56:1036-42.

22. Coppin CM, Gospodarowicz MK, James K, et al. Improved local control of invasive bladder cancer by concurrent cisplatin and preoperative or definitive radiation. The National Cancer Institute of Canada Clinical Trials Group. J Clin Oncol. 1996;14:2901-7.

23. Kasuga I, Makino S, Kiyokawa H, et al. Tumor-related leukocytosis is linked with poor prognosis in patients with lung carcinoma. Cancer. 2001;92:2399-405.

24. Köhne CH, Cunningham D, Di Costanzo F, et al. Clinical determinants of survival in patients with 5 -fluorouracil-based treatment for metastatic colorectal cancer: results of a multivariate analysis of 3825 patients. Ann Oncol. 2002:13:308-17.

25. Maione P, Rossi A, Di Maio M, et al. Tumor-related leucocytosis and chemotherapy-induced neutropenia: linked or independent prognostic factors for advanced non-small cell lung cancer? Lung Cancer. 2009;66:8-14.

26. So KA, Hong JH, Jin HM, et al. The prognostic significance of preoperative leukocytosis in epithelial ovarian carcinoma: a retrospective cohort study. Gynecol Oncol. 2014;132:551-5.

27. Worley MJ, Nitschmann CC, Shoni M, et al. Preoperative leukocytosis imposes an increased risk of recurrence and death among patients with nonendometrioid endometrial carcinoma. Int J Gynecol Cancer. 2013;23:312-7.

28. Chen Y-W, Chen I-L, Lin I-C, et al. Prognostic value of hypercalcaemia and leucocytosis in resected oral squamous cell carcinoma. Br J Oral Maxillofac Surg. 2014;52:425-31.

29 Granger JM, Kontoyiannis DP. Etiology and outcome of extreme leukocytosis in 758 nonhematologic cancer patients. Cancer. 2009;115:3919-23.

30 Cerny J, Rosmarin AG. Why does my patient have leukocytosis? Hemato Oncol Clin North Am. 2012;26:303-19.

\section{Submit your next manuscript to BioMed Central and we will help you at every step:}

- We accept pre-submission inquiries

- Our selector tool helps you to find the most relevant journal

- We provide round the clock customer support

- Convenient online submission

- Thorough peer review

- Inclusion in PubMed and all major indexing services

- Maximum visibility for your research

Submit your manuscript at www.biomedcentral.com/submit
Biomed Central 